\title{
Design of Modular Remote Monitoring System for Low Temperature
}

\section{Environment}

\author{
Mei-lian Zhao ${ }^{1, a,{ }^{*}, \text { Hai-chun Niu }}{ }^{1, b}$ and Zhi-qiang Liu ${ }^{1, c}$ \\ ${ }^{1}$ Qingdao Huanghai College, Qingdao, Shandong, China \\ a728395038@qq.com, b5 582530799@qq.com, ㄷ150092185@qq.com
}

\begin{abstract}
This paper designs a low-temperature environment modular remote monitoring system. Through the host computer the system can achieve a variety of information configuration, history query, curve report printing and a series of functions. In this paper, the background of system design is introduced, and the hardware and software design of the system is described.
\end{abstract}

Keywords: Low temperature; Modular; Remote monitoring and control.

\section{Introduction}

The state more and more attention to the monitoring of low temperature environment, the business of pharmaceutical enterprises, hospitals, food companies put forward GSP and GMP certification requirements, the requirements of the environment to do a detailed record of temperature records. This request has been launched, and quickly triggered the R \& D enthusiasm of enterprises, all enterprises for the country's requirements, the monitoring of low-temperature environment developed the relevant equipment, more common foreign brands Omega, the domestic brand Ze large, These devices have the following shortcomings: 1.almost no equipment for ultra-low temperature environment, most of the equipment can only be detected minus ten degrees, ultra-low temperature detection of this part is a blank; 2.most of the equipment accuracy 3.most of the equipment is only suitable for small-scale point-to-point monitoring, if the cold storage after the need for sub-regional monitoring, not self-organizing network.4.the equipment is relatively simple, not according to the scene to quickly establish the application system. With the development of ZigBee technology, 4G technology, sensor technology and other technologies mature, to achieve the entire low-temperature environment, information collection, automation, modular, must be the next step in the development trend.

\section{System composition and working principle}

Low temperature environment Modular remote monitoring system consists of terminal node, sink node, host computer composed of three parts. The terminal node collects the information of the low temperature environment through the sensor or the sensing circuit and sends the collected information to the sink node through the wired 485 , the wireless $433 \mathrm{M}$ communication and the ZigBee networking, etc. The sink node module can complete the display of the terminal acquisition information, Alarm and through the 4G, cable 485, Internet, etc. to the information sent to the host computer module, the host computer can achieve a 
variety of information configuration, history query, curve report printing and a series of functions. The system block diagram is shown in Figure 1

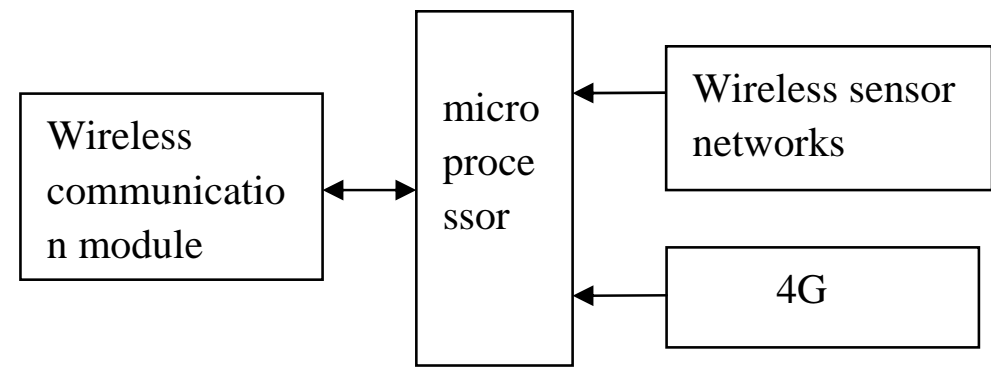

terminal node

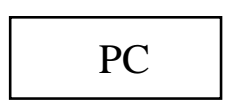

upper machine

Figure 1 system block diagram

\section{System hardware design and implementation}

The hardware is mainly composed of the terminal node module and the sink node module.

Terminal node module. Terminal node module mainly includes temperature sensor, microprocessor and wireless transmitter chip, as shown in Figure 2. System selection PT100 as a temperature sensor, using Atmel's ATmegal28L microcontroller as the microprocessor circuit of this module, using Chipcon's CC2420 RF transceiver as the main transmitter of the wireless transmitter and receiver module. The system requires 4-channel signal sampling temperature, the signal is collected by the sensor through the $\mathrm{A} / \mathrm{D}$ conversion process, the environmental monitoring system real-time temperature data transmission to the convergence node module.

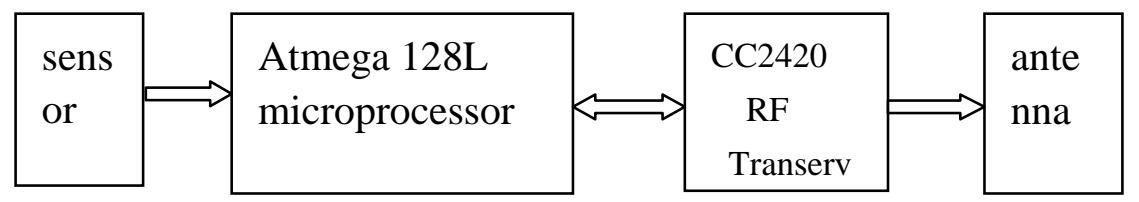

Figure 2 temperature acquisition module node schematic diagram

Temperature sensor circuit. ATmegal28L is an 8-bit microcontroller, the AVR series is currently the most powerful controller. Microprocessor ATmega128L internal AD conversion circuit, and the temperature sensor PT100 connection shown in Figure 3. The resistor R2 in the figure is a conventional voltage limiting resistor to prevent overvoltage or overcurrent to protect the circuit; the resistor $\mathrm{Rl}$ is a thermistor.

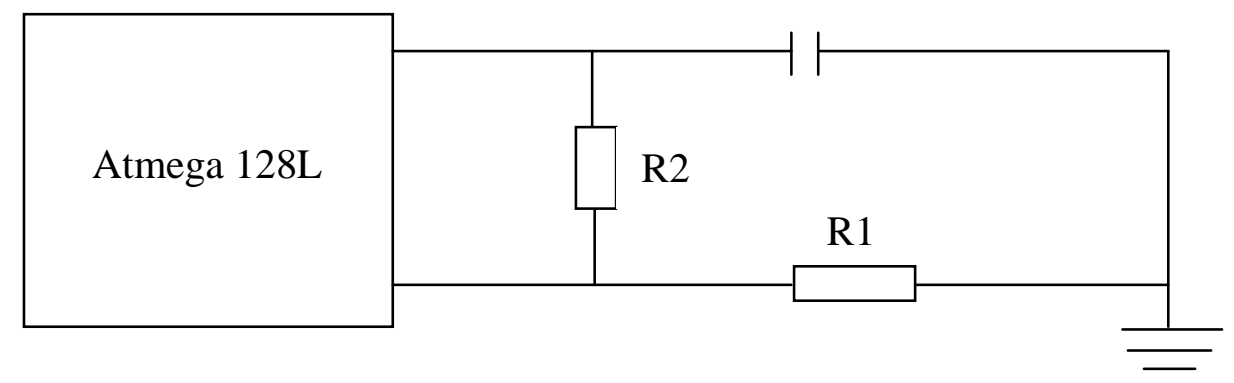

Figure 3 ATmegal128L connection diagram and temperature sensor

Wireless communication module circuit CC2420 chip is in line with 2.4GHZ IEEE802.15.4 standard RF transceiver, which is based on Chipcon's SmartRF03 technology only a few external components that achieve stable performance, and low power consumption. CC2420 
and ATmega128L processor connection is relatively simple, and CC2420 exchange data, send command, ATmega128L processor mainly through the 4-wire SPI bus (SO, CSn, SCLK, SI) to set the working mode; use RESET pin to reset the chip. ATmega128L microcontroller 4 SPI interface: SS, MISO, SCLK, MO-SI, corresponding to the CC2420 wireless transceiver chip 4 SPI interface: CSn, SO, SCLK, SI. Wireless transceiver chip CC2420 and A Tmegal 128L microcontroller connection diagram shown in Figure 4.

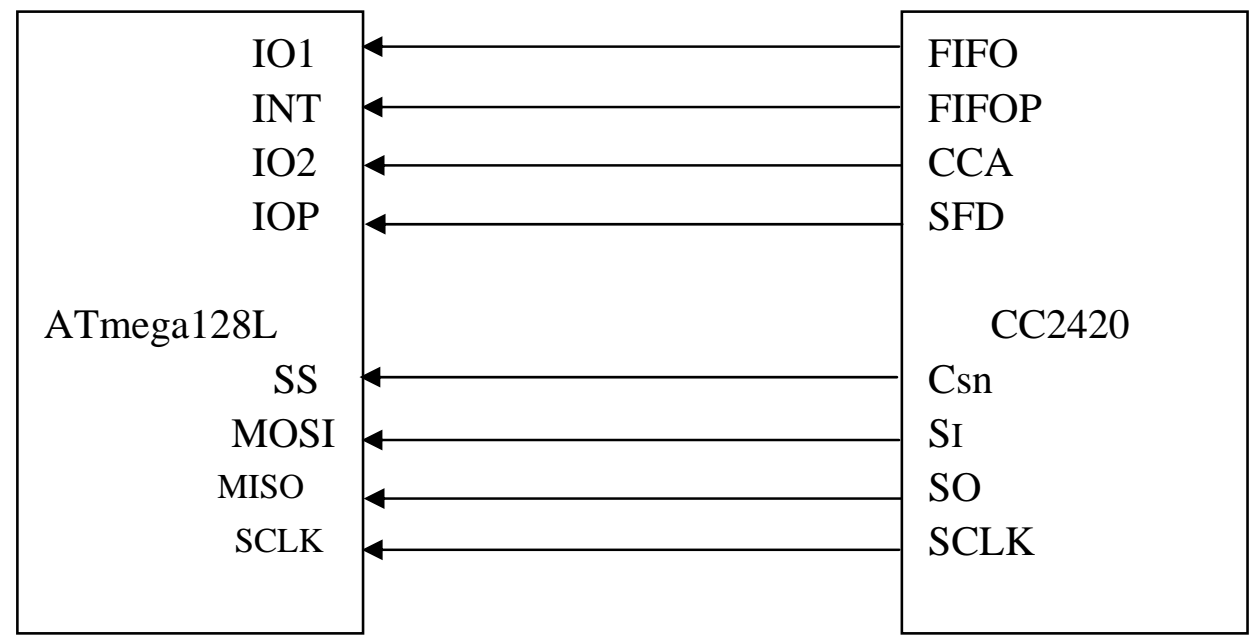

Figure 4 CC2420 and ATmegal128L connection diagram

Gathering node module. The convergence node acts as a bridge between the ZigBee coordinator node and the local area network. It processes the received signal. The processing method mainly passes through the low noise amplifier and the quadrature downconversion into the intermediate frequency signal, then converts it into digital signal, To connect the temperature value to the host computer. Users can obtain the temperature value of each node of the system through the host computer, realize the remote monitoring and control of the temperature, and can set the parameter value when the alarm is needed. The block diagram of its convergence node is shown in Figure 5.

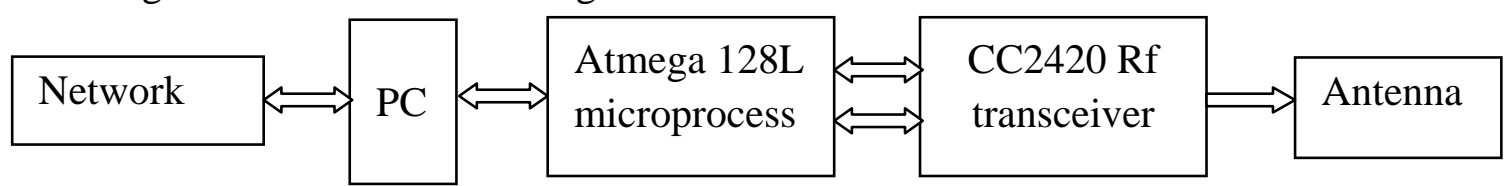

Figure 5 combine the principle block diagram of the node

\section{System software design}

Design of terminal acquisition node software The main work of the sensor terminal node is data information collection, network connection and take part of the routing function, to maintain communication with the neighboring nodes, detection of link status. Through the wireless ad hoc network will be real-time accurate collection of temperature data sent to the server for storage and analysis.

Design of terminal control node software. The terminal node realizes the temperature information in real time, uses ZigBee technology to transmit the information to the gateway, and the gateway processor transmits the information to the host computer through the 4G, and the host computer can realize the information configuration, the historical record inquiry and the curve report printing function. The workflow of the whole system is shown in Figure 6. 


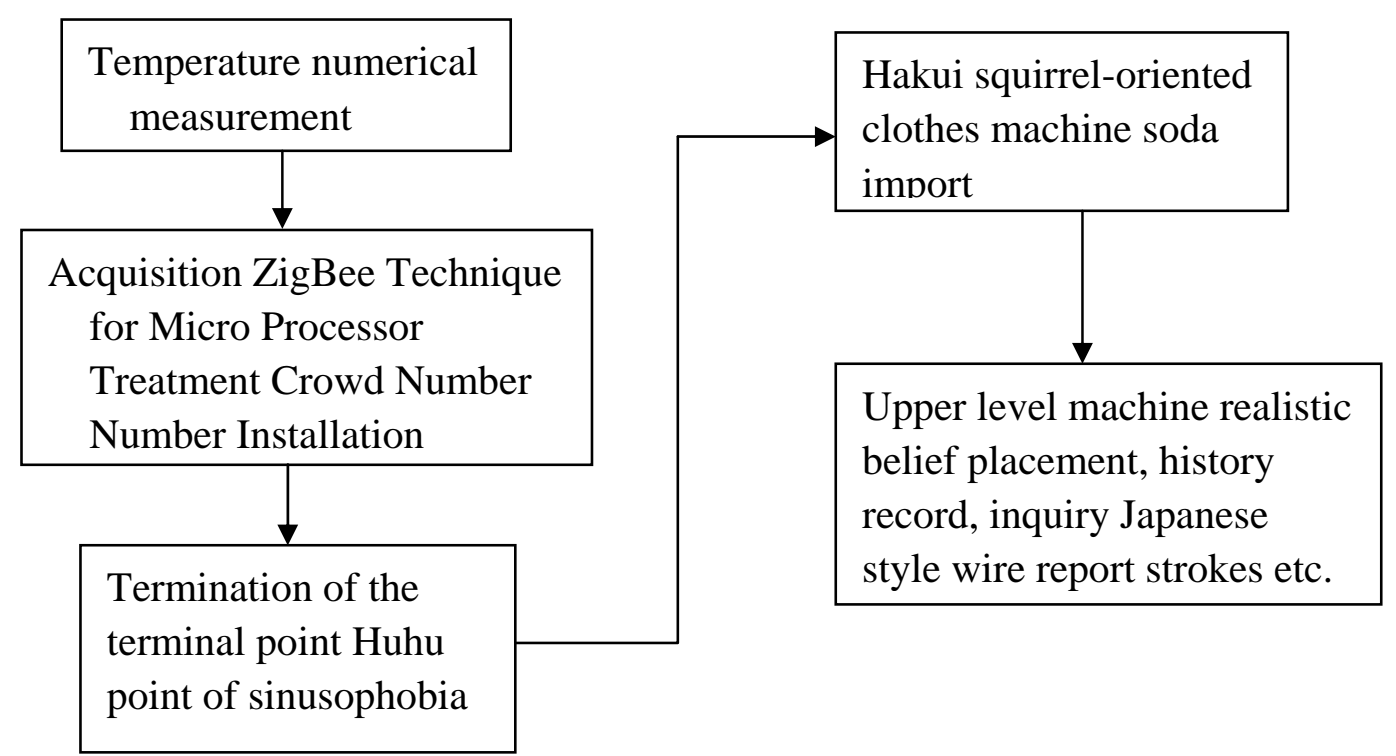

Figure 6 system work flow chart

\section{Conclusion}

Starting from the design of the terminal node and the convergence node of the remote monitoring system of the low temperature environment and the realization way of each module, a kind of real-time monitoring method of cold chain temperature is designed, which is effective, convenient and inexpensive. The corresponding software system summarizes the monitoring data collected by the temperature acquisition module from each node and analyzes the temperature change in the cold chain equipment according to the temperature limit of the product set by the system. If the abnormality and abnormality are found, The system will be through a variety of communication mechanisms, issued pre-alarm information. The system has real-time, accurate, convenient, reliable and so on.

\section{Reference}

[1] Xu Fengliang Wang Fenglin, yi-huai wang, etc. The monitoring system based on wireless sensor network and $3 \mathrm{~g}$ research and application [J]. Journal of measurement and control technology, 2013, 32 (8) : 99-102.

[2] ZhangWeiCong YuXinWu, lee choong-sung. Based on CC2530 and ZigBee protocol stack design wireless sensor nodes [J]. Computer system application, 2011, 20 (7) : 184-187

[3] Hong-jun wang. Cold chain temperature monitoring system based on wireless sensor network research [D]. Harbin university of science and technology, 2011

[4] Xi-feng liu. Embedded refrigerator car environment remote monitoring system design based on ZigBee [D]. Gansu agricultural university, 2013 